\title{
乙基溴化镁与二异丙基酮反应的计算化学研究
}

黄职钱, 杨一荣, 马玉臣 ${ }^{*}$ 张冬菊*

山东大学化学与化工学院, 济南 250100

摘要: 格氏试剂与羰基化合物的亲核加成反应是构建 $\mathrm{C}-\mathrm{C}$ 键的经典反应, 该反应通常伴生的副反应主要包括羰基化 合物的还原反应、烯醇化反应等。以乙基澳化镁 $(\mathrm{EtMgBr})$ 与二异丙基酮 $\left(\mathrm{CO}(\mathrm{iPr})_{2}\right)$ 的反应为例, 通过量子化学计算研 究了格氏试剂与嶡基化合物的亲核加成反应、还原反应和烯醇化反应。结果表明, 通过Schlenk平衡形成的二乙基美 $\left(\mathrm{MgEt}_{2}\right)$ 比EtMgBr更容易与 $\mathrm{CO}(\mathrm{iPr})_{2}$ 反应, 与教科书中定性描述的反应机理明显不同; 乙醚溶剂对反应性能有重要影 响, 特别是更有利于亲核加成反应; 计算的三个反应的能垒差可以合理解释实验所观察的主产物与副产物的产率比。

关键词: 格氏试剂; 羊基化合物; 亲核加成; 还原; 烯醇化

中图分类号: G64; O6

\section{Computational Chemistry on Reactions of Ethylmagnesium Bromide with Diisopropyl Ketone}

\author{
Zhiqian Huang, Yiying Yang, Yuchen Ma *, Dongju Zhang * \\ School of Chemistry and Chemical Engineering, Shandong University, Jinan 250100, China.
}

Abstract: The nucleophilic addition of Grignard reagents with carbonyl compounds is a classical reaction to construct $\mathrm{C}-\mathrm{C}$ bonds, which is frequently accompanied by side-reactions, mainly including the enolization and reduction of carbonyl compounds. To explore the molecular mechanism of these three reactions, quantum chemical calculations were performed on the reaction of ethylmagnesium bromide $(\mathrm{EtMgBr})$ with diisopropyl ketone $\left(\mathrm{CO}(\mathrm{iPr})_{2}\right)$, a prototypical reaction of Grignard reagents with carbonyl compounds. It is found that diethyl magnesium ( $\left.\mathrm{MgEt}_{2}\right)$ obtained from the Schlenk equilibrium of $\mathrm{EtMgBr}$, is more active toward $\mathrm{CO}(\mathrm{iPr})_{2}$ than $\mathrm{EtMgBr}$, which is generally described as the virtual active species in the textbook. The diethylether is shown to play a substantial role in the reactivity, especially in favor of nucleophilic addition reaction. The calculated energy barriers of the three reactions can rationalized the experimentally observed main product to by-products yield ratio.

Key Words: Grignard reagents; Carbonyl compounds; Nucleophilic addition; Reduction; Enolization

格氏试剂(Grignard reagent)是一类有机镁卤化物的统称, 通常表示为R-MgX, 由法国化学家维 克多·格林尼亚(Victor Grignard)在1900年发现 ${ }^{[1]}$ 。格氏试剂的出现, 在有机合成领域具有里程碑的意

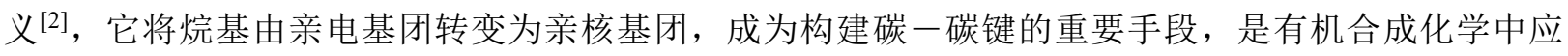
用最多的有机化学试剂之一, 意义重大、影响深远, 被列入基础有机化学教科书, 其发明人格林尼 亚因此获得了1912年诺贝尔化学奖。

收稿: 2021-08-01; 录用: 2021-09-07; 网络发表: 2021-10-25

“通讯作者, Emails: myc@sdu.edu.cn (马玉臣); zhangdj@sdu.edu.cn (张冬菊)

基金资助: 国家自然科学基金面上项目(21773139,21833004); 山东省自然科学基金青年基金项目(ZR2020QB077) 
格氏试剂中 $\mathrm{Mg}-\mathrm{C}$ 键是强极性共价键, 富电子的碳具有很强的碱性和亲核性, 化学性质极为活 泼。格氏试剂最典型的反应是与羰基化合物的亲核加成反应, 形成醇镁盐, 经水解后得到相应的 醇。该反应可以在羰基碳原子上引入一个烃基, 使醛酮碳链延长, 成为合成各种复杂的伯、仲、叔 醇的重要策略。格氏试剂与嶡基化合物除了发生亲核加成反应((1)式)之外, 还涉及一些竞争的副反 应, 主要包括羰基化合物的还原反应((2)式)和烯醇化反应((3)式)等, 这些副反应的存在是导致主产 物产率不够理想的关键因素 ${ }^{[3]}$ 。

尽管格氏试剂的使用已有 100 多年的历史, 但由于其结构的复杂性致使人们对其反应机理的认识 仍不够清晰, 缺乏共识 ${ }^{[3]}$ 。教科书 ${ }^{[4]}$ 中对这些反应的介绍, 仅是简单地进行定性描述, 如图 1 所 示, 用格氏试剂的化学式R-MgX描述其与醛酮的反应机理, 缺乏详细的定量信息。

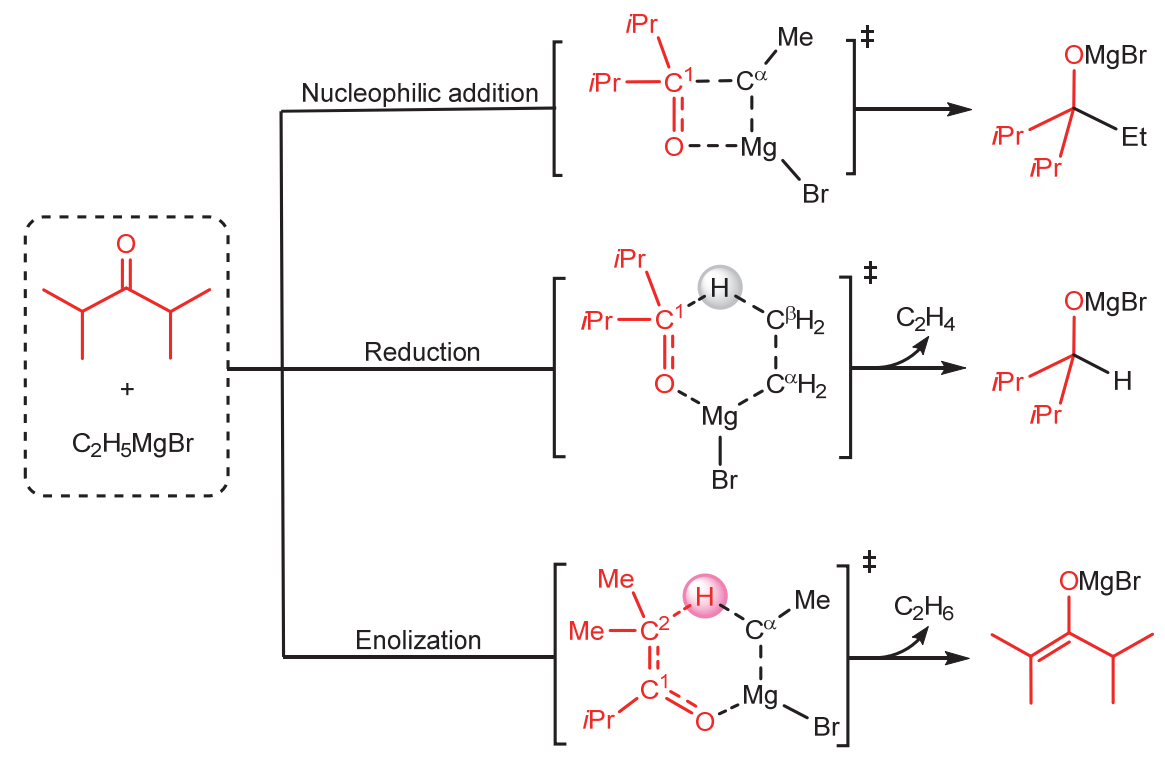

图1 $\mathrm{EtMgBr}$ 和 $\mathrm{CO}(i \mathrm{Pr})_{2}$ 的亲核加成反应、还原反应和烯醇化反应

最近, Eisenstein和Cascella课题组通过量子化学计算和从头算分子动力学模拟研究了甲基氯化镁 格氏试剂 $\left(\mathrm{CH}_{3} \mathrm{MgCl}\right)$ 与乙醛和芴酮的加成反应 ${ }^{[5]}$, 获得了一些关于反应机理的新结果, 在学术界引起 了较大反响, “phys.org” 网站以 “The 120-year old cold case for the Grignard reaction is finally solved” 为题, 对该工作进行了专题报道 ${ }^{[6]}$; “高分子科学前沿” (入选 “2020学术公众号100强”) 媒体号也以 “诺奖 “格氏试剂”之谜终获解! 或将更新教科书? ”为题, 对该工作进行了详细介 绍 ${ }^{[7]}$ 。Eisenstein和Cascella等研究者的主要发现是, 格氏试剂在四氢呋喃溶剂中由于Schlenk平衡 ${ }^{[8]}$ ((4)式)的存在, 可通过多种形式同时与醛酮发生亲核加成反应, 四氢呋喃溶剂对反应有明显的促进 作用。

$$
2 \mathrm{EtMgBr} \rightleftharpoons \mathrm{MgEt}_{2}+\mathrm{MgBr}_{2}
$$

Eisenstein和Cascella课题组的最新研究成果, 改进了人们对格氏试剂与羰基化合物亲核加成反应 的理解。文献调研表明, 对于格氏试剂与羰基化合物的还原反应和烯醇化反应, 尚未有公开报道的 理论研究。本文以(1)-(3)式所示的反应为例, 通过量子化学计算系统研究乙基溴化镁和二异丙基酮 的反应, 以期澄清反应的分子机理, 定量描述反应的动力学和热力学性质, 加深对格氏试剂与羰基 化合物反应性能的理解。

\section{1 模型和计算方法}

如(1)-(3)式所示, 研究对象为乙基溴化镁 $(\mathrm{EtMgBr})$ 与二异丙基酮 $\left(\mathrm{CO}(i \mathrm{Pr})_{2}\right)$ 的反应, 溶剂为乙 
醚 ${ }^{[9]}$ 。根据(4)式所示的 Schlenk平衡, $\mathrm{EtMgBr}$ 和 $\mathrm{MgEt}_{2}$ 在乙醚溶剂中平衡共存, 因此, 计算考虑了 $\mathrm{EtMgBr}$ 和 $\mathrm{MgEt}_{2}$ 两种组分的有机镁与 $\mathrm{CO}(i \mathrm{Pr})_{2}$ 的反应。为便于描述, 将 $\mathrm{CO}(i \mathrm{Pr})_{2}$ 中羰基 $\mathrm{C}$ 原子和参与 反应的三级 $\mathrm{C}$ 原子分别标记为 $\mathrm{C}^{1}$ 和 $\mathrm{C}^{2}, \mathrm{EtMgBr}$ 中亚甲基 $\mathrm{C}$ 原子和甲基 $\mathrm{C}$ 原子分别标计为 $\mathrm{C}^{\alpha}$ 和 $\mathrm{C}^{\beta}$, 如 图1所示。

本研究基于密度泛函理论，使用 M062X泛函 ${ }^{[10]}$ 和6-311G $(d, p)$ 标准基组 ${ }^{[11]}$ 对全部稳定点进行了结 构优化和频率计算, 确保局域最小点没有虚频, 过渡态(一级鞍点)有且仅有一个虚频, 并通过内禀 反应坐标(IRC) ${ }^{[12]}$ 分析, 确认了每个过渡态连接的反应物和产物。应用PCM连续介质模型 ${ }^{[13]}$ 评估了 溶剂化效应, 在乙醚溶剂中对稳定点进行了全构型优化。全部计算使用Gaussian 16程序 ${ }^{[14]}$ 完成。

\section{2 结果与讨论}

\section{1 乙基溴化镁和二异丙基酮的反应}

非显含溶剂情况下, 计算的反应机理及势能面上各稳定点的相对能量示于图 $2 \mathrm{a}$ 。反应从 $\mathrm{EtMgBr}$

(a) $\underset{\substack{\Delta \mathrm{G}_{\text {sol }} \\ \mathrm{kJ} / \mathrm{mol}}}{\mathrm{s}}$

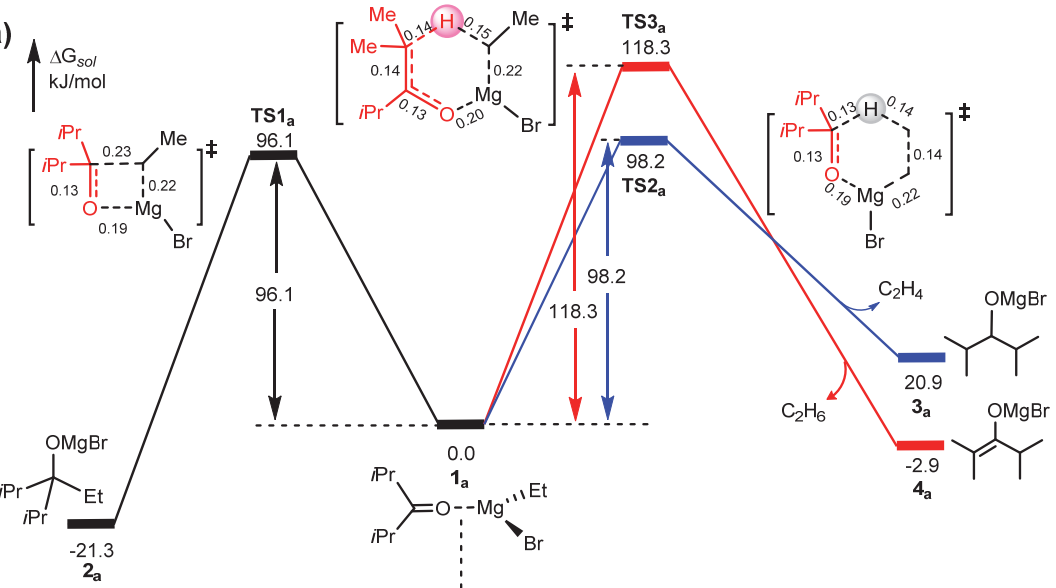

(b)

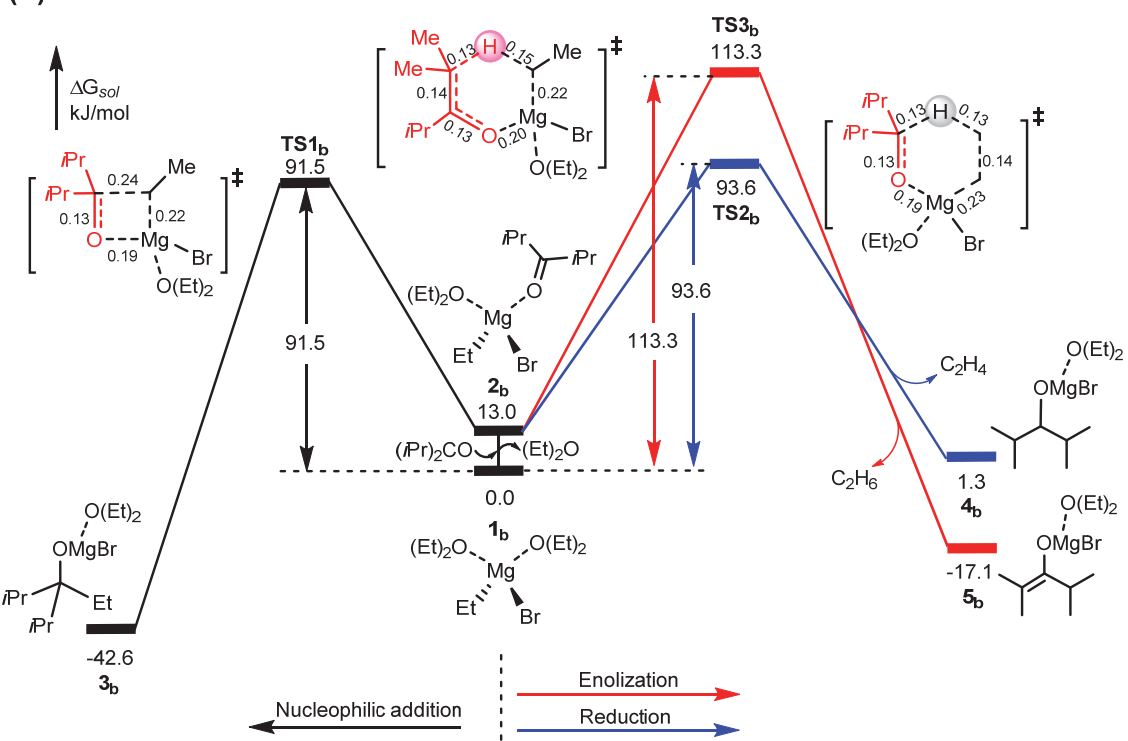

图2 $\mathrm{EtMgBr}$ 与 $\mathrm{CO}(i \mathrm{Pr})_{2}$ 发生亲核加成(黑色)、还原反应(蓝色)和烯醇化反应(红色)的势能剖面图

(a) 非显含溶剂, (b) 显含溶剂。键长: $\mathrm{nm}$ 
与 $\mathrm{CO}(i \mathrm{Pr})_{2}$ 的配位开始, 形成络合物 $\mathbf{1}_{\mathrm{a}}$, 放热 $43.1 \mathrm{~kJ} \cdot \mathrm{mol}^{-1}$, 将该络合物的相对能量设置为参考点。图 中黑色路径表示格氏试剂与嶡基化合物的亲核加成反应, $\mathrm{EtMgBr}$ 的 $\mathrm{C}^{\alpha}$ 亲核进攻 $\mathrm{CO}(i \mathrm{Pr})_{2}$ 的 $\mathrm{C}^{1}$, 同时 羰基 $\mathrm{O}$ 进攻 $\mathrm{Mg}$, 形成四元环状过渡态 $\mathbf{T S 1}$, 其相对能量为 $96.1 \mathrm{~kJ} \cdot \mathrm{mol}^{-1}$, 得到醇镁盐产物 $\mathbf{2}_{\mathbf{a}}$, 放 热 $21.3 \mathrm{~kJ} \cdot \mathrm{mol}^{-1}$ 。蓝色路径表示羰基化合物的还原反应, $\mathrm{EtMgBr}$ 上的 $\mathrm{C}^{\beta}-\mathrm{H}$ 迁移到 $\mathrm{CO}(i \mathrm{Pr})_{2}$ 的 $^{1}$ 上, 同 时羰基 $\mathrm{O}$ 进攻 $\mathrm{Mg}$, 形成六元环过渡态 $\mathbf{T S 2} \mathbf{2}_{\mathbf{a}}$, 克服 $98.2 \mathrm{~kJ} \cdot \mathrm{mol}^{-1}$ 的能垒, 形成还原产物 $\mathbf{3}_{\mathbf{a}}$ 和乙烯, 吸 热 $20.9 \mathrm{~kJ} \cdot \mathrm{mol}^{-1}$ 。红色路径表示烯醇化过程, $\mathrm{CO}(i \mathrm{Pr})_{2}$ 中的 $\mathrm{C}^{2}-\mathrm{H}$ 迁移到 $\mathrm{EtMgBr}$ 的 $\mathrm{C}^{\alpha}$ 上, 同时羰基 $\mathrm{O}$ 进 攻 $\mathrm{Mg}$, 形成六元环状过渡态 $\mathbf{T S 3} 3_{\mathbf{a}}$, 该过程需克服 $118.3 \mathrm{~kJ} \cdot \mathrm{mol}^{-1}$ 的能垒, 得到烯醇化产物 $\mathbf{4}_{\mathbf{a}}$ 和乙烷, 放热 $2.9 \mathrm{~kJ} \cdot \mathrm{mol}^{-1}$ 。这些结果表明, 烯醇化反应显然是不利的, 似乎可以解释实验观察到的极少量烯 醇化产物, 但亲核加成和还原过程的能垒仅相差 $2.1 \mathrm{~kJ} \cdot \mathrm{mol}^{-1}$, 是相互竞争的反应, 这与实验结果不

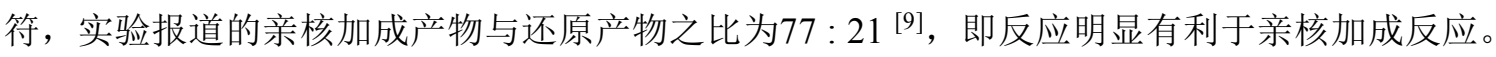

根据Eisenstein和Cascella的研究 ${ }^{[5]}$, 溶剂对反应有重要影响, 因此进一步考虑了显含溶剂的情 况。计算发现, $\mathrm{EtMgBr}$ 与乙醚 $\left(\mathrm{Et}_{2} \mathrm{O}\right)$ 分子的配位是一个放热过程, 其中两分子 $\mathrm{Et}_{2} \mathrm{O}$ 配位形成四面体 配合物是热力学最有利的过程, 放热 $88.9 \mathrm{~kJ} \cdot \mathrm{mol}^{-1}$, 如(5)式所示。

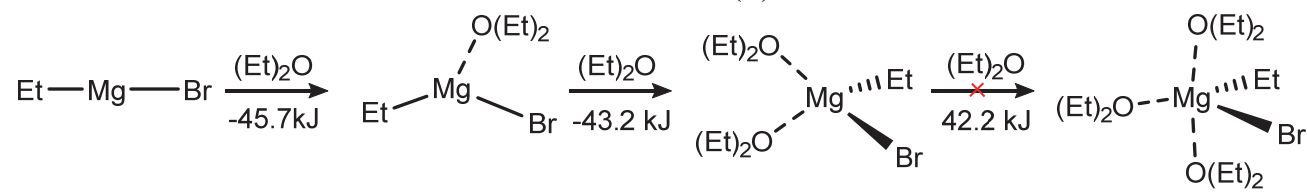

显含溶剂的计算结果示于图 $2 b$, 将两分子 $\mathrm{Et}_{2} \mathrm{O}$ 配位的格氏试剂 $\mathbf{1}_{\mathrm{b}}$ 设置为能量参考点。反应从配 体交换开始, $\mathrm{CO}(i \mathrm{Pr})_{2}$ 取代一个 $\mathrm{Et}_{2} \mathrm{O}$ 分子, 形成中间体 $\mathbf{2}_{\mathbf{b}}$, 吸热 $13.0 \mathrm{~kJ} \cdot \mathrm{mol}^{-1}$ 。 $\mathbf{2}_{\mathbf{b}}$ 分别经过 $\mathbf{T S 1}_{b} 、 T S 2_{b} 、 T S 3_{b}$ 发生亲核加成、还原反应和烯醇化反应, 从计算的过渡态结构参数, 可以看 出, $\mathrm{Et}_{2} \mathrm{O}$ 分子在反应中是形式上的 “旁观者” , 相应的能垒分别为 $91.5 、 93.6$ 和 $113.3 \mathrm{~kJ} \cdot \mathrm{mol}^{-1}$, 这些 结果与图1中未考虑溶剂参与反应的情况相比, 能垒降低约 $5 \mathrm{~kJ} \cdot \mathrm{mol}^{-1}$, 但三个反应的相对能垒定性 没有变化, 例如, $\mathbf{T S 1}_{\mathbf{b}}$ 与 $\mathbf{T S 2} \mathbf{2}_{\mathbf{b}}$ 的能垒依然仅相差 $2.1 \mathrm{~kJ} \cdot \mathrm{mol}^{-1}$ 。

\section{2 二乙基美和二异丙基酮的反应}

如(4)式所示, 格氏试剂在溶剂中存在 Schlenk平衡, 乙基澳化镁 $\mathrm{EtMgBr}$ 与二烷基镁 $\mathrm{MgEt}_{2}$ 共 存。 $\mathrm{MgEt}_{2}$ 中的烷基类似于格氏试剂中的烷基, 具有强亲核性。因此, 我们以 $\mathrm{MgEt}_{2}$ 和 $\mathrm{CO}(i \mathrm{Pr})_{2}$ 为反 应底物, 重新对上述三个反应进行了计算, 结果示于图 3 , 其中图 $3 \mathrm{a}$ 和 $3 \mathrm{~b}$ 分别描述非显含溶剂和显含 溶剂的情况。

非显含溶剂时, 将 $\mathrm{MgEt}_{2}$ 与 $\mathrm{CO}(i \mathrm{Pr})_{2}$ 的配位物 $\mathbf{1}_{\mathbf{c}}$ 设置为能量参考点。亲核加成、还原反应和烯醇 化反应分别经过 $\mathbf{T S 1}$ 、 $\mathbf{T S 2}_{\mathbf{c}}$ 和 $\mathbf{T S 3} \mathbf{c}_{\mathbf{c}}$ 得到相应的产物, 对应的能垒分别为 $81.9 、 85.7$ 和 $99.9 \mathrm{~kJ} \cdot \mathrm{mol}^{-1}$ 。与 $\mathrm{EtMgBr}$ 的反应(图2a)相比, 能垒降低约12-18 $\mathrm{kJ} \cdot \mathrm{mol}^{-1}$ 。

显含溶剂时, 三个反应的能垒分别进一步降低到69.0、76.9和 $97.0 \mathrm{~kJ} \cdot \mathrm{mol}^{-1}$, 与EtMgBr对应的三 个反应(图2b)相比能垒更低, 特别是更有利于亲核加成反应, 使其能垒比还原反应低 $7.9 \mathrm{~kJ} \cdot \mathrm{mol}^{-1}$ 。

将图3与图2比较, 可以发现, $\mathrm{MgEt}_{2}$ 比 $\mathrm{EtMgBr}$ 更容易与 $\mathrm{CO}(i \mathrm{Pr})_{2}$ 发生反应, 反应的能垒更低, 放 热更多。这些结果区别于教科书中对反应机理的定性描述(图1)。另外, 对于本文研究的反应体 系, 根据阿伦尼乌斯公式, 无论用 $\mathrm{EtMgBr}$ 或 $\mathrm{MgEt}_{2}$ 作为反应底物, 无论非显含溶剂或显含溶剂的反 应, 均是亲核加成反应最为有利, 其次是还原反应, 烯醇化反应的能垒最高, 不能与亲核加成和 还原反应竞争。这些结果与实验观察基本吻合, 实验发现亲核加成产物产率为 $77 \%$, 还原产物产 率为 $21 \%$ ，而烯醇化产物产率仅为 $2 \%{ }^{[9]}$ 。

\section{3 烷基取代基和溶剂对反应性能的影响}

如上所述, $\mathrm{MgEt}_{2}$ 比 $\mathrm{EtMgBr}$ 更容易与 $\mathrm{CO}(i \mathrm{Pr})_{2}$ 反应, 并且 $\mathrm{Et}_{2} \mathrm{O}$ 溶剂在 $\mathrm{Mg}$ 中心的配位使反应的能 垒进一步降低。这些结果与取代基及醚配体的电子性质有关, $\mathrm{EtMgBr}$ 中 Br是吸电子取代基, 而 $\mathrm{MgEt}_{2}$ 中Et 是给电子取代基、 $\mathrm{Et}_{2} \mathrm{O}$ 是富电子配体, 上述三个反应均涉及 $\mathrm{Mg}-\mathrm{C}$ 键的异裂, 给电子取代 
基( $\mathrm{Et})$ 和富电子配体 $\left(\mathrm{Et}_{2} \mathrm{O}\right)$ 配体有利于 $\mathrm{Mg}-\mathrm{C}$ 键的极化和异裂, 从而使反应更容易进行。为定量理解 烷基取代基和溶剂对反应性能的影响, 进一步计算了 $\mathrm{MgEt}_{2}$ 和 $\mathrm{EtMgBr}$ 中 $\mathrm{Mg}-\mathrm{C}$ 键异裂过程的焓 变, 比较了 $\mathrm{Et}_{2} \mathrm{O}$ 配位数对焓变的影响, 结果列于表 1 。可以看出, 当底物相同时, 随着 $\mathrm{Et}_{2} \mathrm{O}$ 溶剂配位 数的增加, $\mathrm{Mg}-\mathrm{C}$ 键异裂的解离能显著降低; 当 $\mathrm{Et}_{2} \mathrm{O}$ 配位数相同时, $\mathrm{EtMgBr}$ 中 Mg $-\mathrm{C}$ 键的解离能明 显高于 $\mathrm{MgEt}_{2}$ 中 $\mathrm{Mg}-\mathrm{C}$ 的键解离能。因此, $\mathrm{MgEt}_{2}$ 中的 $\mathrm{Mg}-\mathrm{C}$ 键较弱, 更容易断裂, 反应能垒更 低, 反应更容易进行。
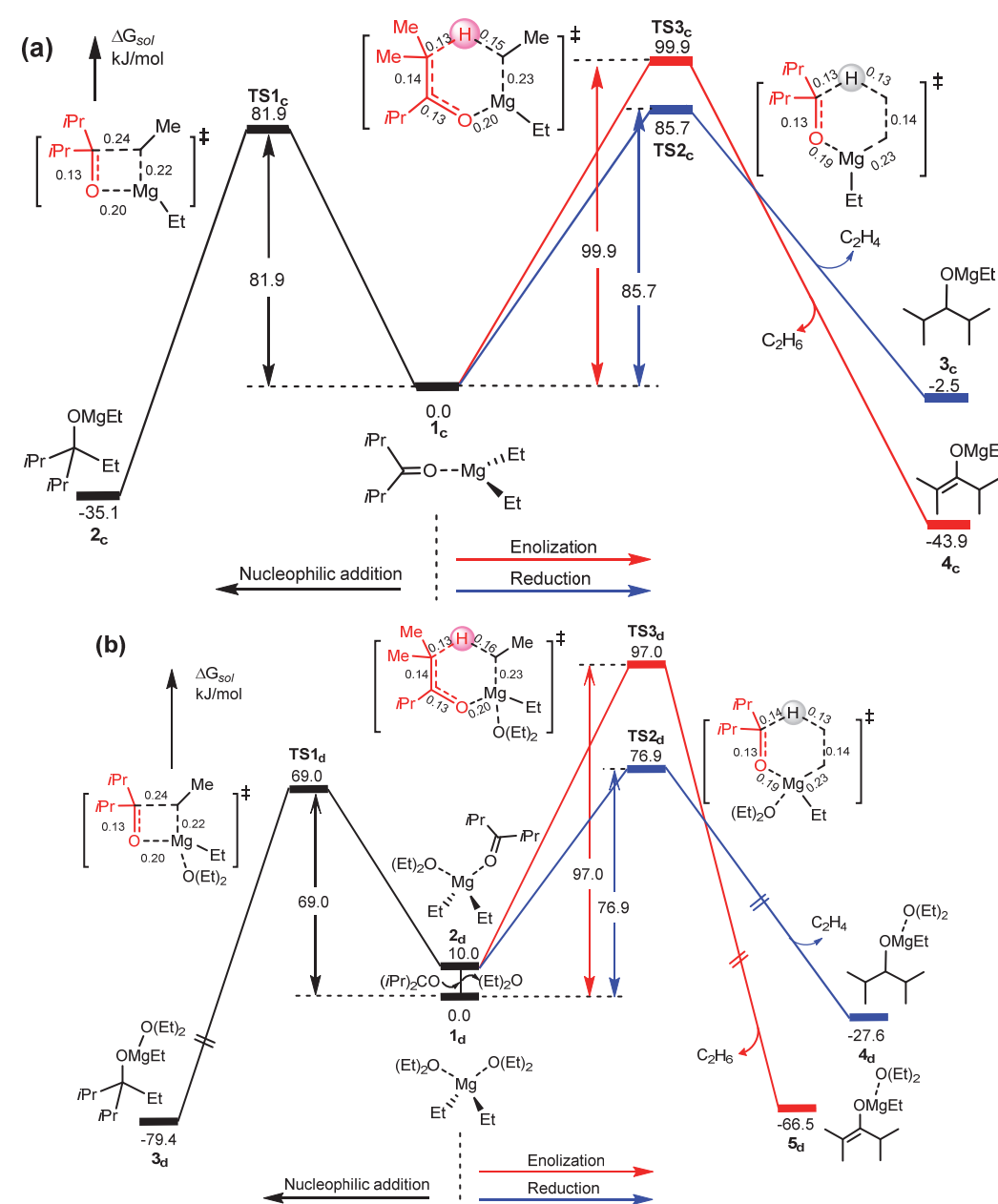

图3 $\mathrm{EtMgBr}$ 与 $\mathrm{CO}(\mathrm{iPr})_{2}$ 发生亲核加成(黑色)、还原反应(蓝色)和烯醇化反应(红色)的势能剖面图

(a) 未考虑显含溶剂, (b) 显含溶剂。键长: nm 电子版为彩图

表1 $\mathrm{EtMgBr}$ 和 $\mathrm{Mg}(\mathrm{Et})_{2}$ 中 $\mathrm{Mg}-\mathrm{C}$ 键异裂的键解离能

\begin{tabular}{ccc}
\hline 底物 & 乙梄配位数 & 键解离能 $\left(\mathrm{kJ} \cdot \mathrm{mol}^{-1}\right)$ \\
\hline $\mathrm{EtMgBr}$ & 0 & 479.4 \\
& 1 & 436.8 \\
& 2 & 392.7 \\
\hline $\operatorname{Mg}(\mathrm{Et})_{2}$ & 0 & 408.1 \\
& 1 & 372.5 \\
& 2 & 331.9 \\
\hline
\end{tabular}




\section{3 结语}

基于密度泛函理论计算，系统研究了乙基溴化镁和二异丙基酮的亲核加成反应、还原反应和烯 醇化反应。计算结果表明, 相关的反应机理与教科书中描述的反应机理不同, $\mathrm{MgEt}_{2}$ 比 $\mathrm{EtMgBr}$ 具有 更高的反应活性, 是反应的主要活性组分; $\mathrm{Et}_{2} \mathrm{O}$ 溶剂对反应性能有重要影响, 通过与 $\mathrm{Mg}$ 配位促进 $\mathrm{Mg}-\mathrm{C}$ 键极化, 使反应更容易发生; 亲核加成反应最容易发生, 其次为还原反应, 烯醇化反应最困 难。理论结果为深刻理解格氏试剂与羰基化合物的反应提供了一定的理论依据。

\section{参 考 文 献}

[1] Grignard, V. C. Compt. Rend. Hebd. Séances Acad. Sci. 1900, 130, 1322.

[2] Rappoport, Z.; Marek, I.; Eds. The Chemistry of Organomagnesium Compounds; Wiley-VCH: Weinheim, Germany, 2008.

[3] Seyferth, D. Organometallics 2009, 28, 1598.

[4] 邢其毅, 裴伟伟, 徐瑞秋, 裴坚. 基础有机化学. 第4版. 北京: 北京大学出版社, 2016: 457-459.

[5] Eisenstein, O.; Cascella, M.; Gauss, J.; Peltzer, R. M. J. Am. Chem. Soc. 2020, 142, 2984.

[6] The 120-year old cold case for the Grignard reaction is finally solved. [2020-02-07]. https://phys.org/news/2020-02-year-cold-case-grignard-reaction.html

[7] 诺奖“格氏试剂”之谜终获解! 或将更新教科书? [2020-02-18]. http://www.xincailiao.com/news/news_detail.aspx?id=554051

[8] Cascella, M.; Nova, A.; Eisenstein, O.; Peltzer, R. M. J. Phys. Chem. B 2017, 121, 4226.

[9] Whitmore, F. C.; George, R. S. J. Am. Chem. Soc. 1942, 64, 1239.

[10] Thiel, W.; Neese, F.; Breidung, J.; Altun, A. J. Chem. Theory Comput. 2014, 10, 3807.

[11] Krishnan, R.; Binkley, J. S.; Seeger, R.; Pople, J. A. J. Chem. Phys. 2008, 72, 650

[12] Fukui, K. Accounts Chem. Res. 1981, 14, 363.

[13] Tapia, O. J. Math. Chem. 1992, 10, 139.

[14] Frisch, M. J.; Trucks, G. W.; Schlegel, H. B.; Scuseria, G. E.; Robb, M. A.; Cheeseman, J. R.; Scalmani, G.; Barone, V.; Petersson, G. A.; Nakatsuji,H.; et al. Gaussian 16, Revision A. 03; Gaussian, Inc.: Wallingford, CT, USA, 2016. 